\title{
Search for the elusive optical counterpart of PSR J0537-6910 with the HST Advanced Camera for Surveys
}

\author{
R. P. Mignani ${ }^{1}$, L. Pulone ${ }^{2}$, G. Iannicola ${ }^{2}$, G. G. Pavlov ${ }^{3}$, L. Townsley ${ }^{3}$, and O. Y. Kargaltsev ${ }^{3}$ \\ 1 European Southern Observatory, Karl-Schwarzschild-Str. 2, 85748 Garching, Germany \\ e-mail: rmignani@eso.org \\ 2 INAF - Osservatorio Astronomico di Roma, Via di Frascati 33, 00040 Monte Porzio Catone, Italy \\ e-mail: [pulone; giacinto] @mporzio.astro.it \\ 3 Pennsylvania State University, 525 Davey Lab, University Park, PA 16802, USA \\ e-mail: [pavlov; townsley; green]@astro.psu.edu
}

Received 3 August 2004 / Accepted 25 October 2004

\begin{abstract}
We present the results of deep, high-resolution, multi-band optical observations of the field of the young ( $\sim 5000 \mathrm{yr})$ 16 ms X-ray pulsar PSR J0537-6910 performed with the Advanced Camera for Surveys (ACS) aboard the Hubble Space Telescope (HST). Although a few new potential counterparts have been detected within or close to the revised Chandra X-ray error circle $\left(\simeq 1^{\prime \prime}\right)$ of the pulsar, only two of them (with magnitudes $m_{814 \mathrm{~W}} \approx 23.9$ and $m_{814 \mathrm{~W}} \approx 24.2$ ) show indications of a peculiar spectrum which could be related to optical emission from the pulsar. This might be true also for a third, fainter, candidate detected only in one filter (with magnitude $m_{814 W} \approx 26.7$ ). If either of the two brighter candidates is indeed the actual counterpart, the optical output of PSR J0537-6910 would make it similar to young Crab-like pulsars. If not, it would mean that PSR J0537-6910 is significantly underluminous with respect to all pulsars detected in the optical.
\end{abstract}

Key words. stars: pulsars: individual: PSR J0537-6910

\section{Introduction}

PSR J0537-6910 is a young X-ray pulsar at the center of the plerionic supernova remnant $\mathrm{N} 157 \mathrm{~B}$ in the LMC, close to the 30 Doradus star forming region. Pulsations with a $16 \mathrm{~ms}$ period were serendipitously discovered by RXTE (Marshall et al. 1998) and immediately confirmed by ASCA and Beppo-SAX (Cusumano et al. 1998). Thus, PSR J0537-6910 has taken over the Crab pulsar $(P=33 \mathrm{~ms})$ as the fastest "ordinary" pulsar (i.e., not spun up by accretion from a companion star) discovered so far. The time derivative of the pulsar period $(\dot{P} \approx$ $5 \times 10^{-14} \mathrm{~s} \mathrm{~s}^{-1}$ ), obtained from the multi-epoch timing (Marshall et al. 1998; Cusumano et al. 1998) yielded a spin down age of $\sim 5000 \mathrm{yr}$, similar to the age of N157B (Wang \& Gotthelf 1998), which makes PSR J0537-6910 one of the handful of known pulsars younger than $10000 \mathrm{yr}$. At the same time, with a derived rotational energy loss $\dot{E} \approx 4.8 \times 10^{38} \mathrm{erg} \mathrm{s}^{-1}$, it is the most energetic pulsar known. As expected for a young pulsar, large glitches in the spindown have been repeatedly detected during different RXTE timing campaigns (Middleditch et al. 2001; Marshall et al. 2004). Observations of PSR J0537-6910 performed with the Chandra X-ray Observatory (Wang et al. 2001; Townsley et al. 2005) have resolved a compact $\left(\simeq 22^{\prime \prime} 5 \times 7^{\prime \prime}\right)$ pulsar-wind nebula (PWN) elongated perpendicular to the symmetry axis of a larger comet-like structure $\left(\simeq 40^{\prime \prime}\right)$. This structure can be explained as a trail left behind by the pulsar during its motion in the ISM. Although its large energy output makes PSR J0537-6910 a natural target for multiwavelength observations, it has not yet been detected outside the X-ray band. So far, searches for its radio counterpart yielded null results, with an upper limit of $F_{1.4 \mathrm{GHz}} \sim 0.04$ mJy (Crawford et al. 1998), which suggests that its radio luminosity is significantly weaker than those of the Crab pulsar and of the other young LMC pulsar B0540-69. Searches for an optical counterpart of PSR J0537-6910 have also been unsuccessful so far. First observations performed with the ESO NTT (Mignani et al. 2000) and the ESO $3.6 \mathrm{~m}$ (Gouiffes \& Ögelman 2000) telescopes failed to detect any potential pulsar counterpart within the original ROSAT $5^{\prime \prime}$ error circle (Wang \& Gotthelf 1998) down to $V \sim 23.4$. The same conclusion was reached by Butler et al. (2002) using archived HST observations of the field and exploiting the updated Chandra pulsar position (Wang et al. 2001). Here we report on the results of recent, much deeper, HST observations of the PSR J0537-6910 field. The same dataset has been also used to search for the optical counterpart of the X-ray Pulsar Wind Nebula (PWN); the results will be reported elsewhere. The observations and data analysis are described in Sects. 2 and 3, respectively, while the results are presented in Sect. 4 and discussed in Sect. 5 . 


\section{Observations}

The field of PSR J0537-6910 was observed on January 2003 with the Wide Field Channel (WFC) of the Advanced Camera for Surveys (ACS) on board the HST. The ACS/WFC, a twoCCD $4096 \times 2048$ pixel detector (Pavlovsky et al. 2003), has a combined field of view of $3.3 \times 3.3$ and an average pixel size of 0.050 . Observations were performed during two consecutive $H S T$ orbits through the $435 W(\lambda=4297 \AA ; \Delta \lambda=1038 \AA)$, $555 W(\lambda=5346 \AA ; \Delta \lambda=1193 \AA)$ and $814 W(\lambda=8333 \AA$; $\Delta \lambda=2511 \AA$ ) filters, selected to maximize the spectral coverage. For each filter, sequences of four exposures were taken to allow cosmic ray filtering and to minimize saturation effects of bright stars in the field close to the position of our target. The corresponding total integration times were $3200 \mathrm{~s}, 2800 \mathrm{~s}$ and $2800 \mathrm{~s}$ in the $435 \mathrm{~W}, 555 \mathrm{~W}$ and $814 \mathrm{~W}$ filters, respectively. Standard data reduction (debiassing, flatfielding) and photometric calibration were applied according to the standard ACS pipeline. The default ACS photometric calibration available at the time of image acquisition was later updated by using as a reference the new instrument zero-points computed from the improved Detector Quantum Efficiency (DQE) and filter throughput curves (De Marchi et al. 2004). For each filter, the STSDAS drizzle task was used to combine single exposures and produce a mosaic image from the two ACS/WFC chips after correcting for the geometric distortions of the CCDs.

\section{Data analysis}

\subsection{HST ACS astrometry}

The absolute uncertainty of the astrometric solution in the HST focal plane is still based on guide stars from the GSC1.1 (Lasker et al. 1990) and is of the order of 1'.0 (Pavlovsky et al. 2003). For this reason, we reassessed the ACS astrometry using more precise catalogs as a reference. Although the GSC2 ${ }^{1}$ seems to be the first natural choice for this purpose, it has been discarded because of coarser astrometric accuracy in this specific field. This is likely caused by systematics affecting the catalog in the LMC region, related to both the local crowding and the presence of diffuse nebulosities associated with the N157B supernova remnant. On the other hand, the recently released USNO-B1.0 catalog (Monet et al. 2003) seems also to be affected by some kinds of systematics and provides only a poor and uneven coverage of the region, with most of the reference stars located at the edge of the ACS field of view. Therefore, we decided to use the Two Micron All Sky Survey (2MASS) Point Source catalog (Skrutskie et al. 1997). After matching on the ACS images the sky and pixel coordinates of 30 well distributed 2MASS stars, a revised astrometric solution has been computed using the package $\mathrm{ASTROM}^{2}$, yielding an overall accuracy of $\sim 0$. $^{\prime} 1$ per coordinate. As an independent check, we qualitatively evaluated the astrometric solution using as a reference the positions of stars selected from the very accurate (0.'035) UCAC2 catalog (Zacharias et al. 2004). The matches between the actual and expected positions were found to be

\footnotetext{
1 http://wwW-gsss. stsci.edu/gsc/gsc2/GSC2home.htm

2 http://star-www.rl.ac.uk/Software/software.htm
}

consistent within the uncertainty of our astrometric calibration. We note that since the UCAC2 is a relatively bright catalog $(V \leq 16)$, all the matched stars are saturated in the ACS images and cannot be used directly for astrometric calibration.

\subsection{Chandra ACIS astrometry}

As a reference for the search of the pulsar counterpart we use the most precise X-ray positions obtained with Chandra (Weisskopf et al. 2002). The previous Chandra position obtained with the imaging array of the High Resolution Camera (HRC-I) by Wang et al. (2001) has been recently reassessed by improving the aspect solution (Gotthelf 2003, private communication). Independent position determinations have also been obtained through the analysis of ACIS (Advanced CCD Imaging Spectrometer) observations, taken in the ACIS-I (imaging array) and ACIS-S (spectroscopy array) modes (Townsley et al. 2005). The original ACIS-I data (ObsID 62520), taken in September 1999, were centered on the R136 stellar cluster in 30 Doradus. The observations were performed in "alternating" mode consisting of sequences of ten $3.3 \mathrm{~s}$ frames followed by a single $0.3 \mathrm{~s}$ frame to eliminate pile-up in N157B. We used the short frames of the ACIS-I observation integrated over the full energy band $(0.5-8 \mathrm{keV})$ to estimate the pulsar position. Unfortunately, the small number of counts available $(\approx 20)$ prevented us from obtaining the position in hard-band channels, so that counts from the PWN and/or the SNR may be affecting the determination of the actual pulsar centroid. To reduce the uncertainty of the pulsar position, we tried to improve the ACIS-I absolute astrometry by cross-matching the coordinates of 30 serendipitous X-ray sources with the 2MASS catalog, but, unfortunately, found only 4 high-confidence matches, which did not allow us to obtain a boresight correction more precise than $\sim 0$ ! 3 . Systematic uncertainties associated with the relatively large off-axis angle may also affect the absolute position of the pulsar measured from the ACIS-I data. Such systematics have been measured in the Chandra Deep Field North (Alexander et al. 2002) and in Orion (Getman et al. 2005) and at an off-axis angle of $5^{\prime}$ they turn out to be $\approx 0$.' 2 .

Given these caveats, the estimated pulsar position is $\alpha(\mathrm{J} 2000)=05^{\mathrm{h}} 37^{\mathrm{m}} 47^{\mathrm{s}} .46, \delta(\mathrm{J} 2000)=-69^{\circ} 10^{\prime} 19^{\prime} \cdot 9$, with an approximate error of 1 '”, which accounts for both the errors of the pulsar centroid and the uncertainty of our boresight correction. The ACIS-S observation (ObsID 2783) had N157B at the aim point, and it was taken in a subarray mode to minimize pile-up. The pulsar centroid, measured using wavdetect over the full energy band $(0.5-8 \mathrm{keV})$, yields a position of $\alpha(\mathrm{J} 2000)=05^{\mathrm{h}} 37^{\mathrm{m}} 47^{\mathrm{s}} .42, \delta(\mathrm{J} 2000)=-69^{\circ} 10^{\prime} 20^{\prime} .2$. The same procedure, but cutting in the $3-7 \mathrm{keV}$ energy range to minimize contamination from the PWN/SNR background, yields a position of $\alpha(\mathrm{J} 2000)=05^{\mathrm{h}} 37^{\mathrm{m}} 47^{\mathrm{s}} .39, \delta(\mathrm{J} 2000)=-69^{\circ} 10^{\prime} 20^{\prime}$ ' 1 , i.e. consistent with the previous one. The errors on these positions lack the systematics present in the off-axis ACIS-I observation. However, due to the smaller field of view in sub-array mode, too few X-ray sources were detected to compute the boresight correction by cross-matching with $2 \mathrm{MASS}$ or to register the 
Table 1. Chandra coordinates of PSR J0537-6910. In all cases an error radius of $1^{\prime \prime}$ is estimated (see text).

\begin{tabular}{llll}
\hline \hline Source & $\begin{array}{l}\alpha(\mathrm{J} 2000) \\
(\mathrm{h} \mathrm{m} \mathrm{s})\end{array}$ & $\begin{array}{l}\delta(\mathrm{J} 2000) \\
\left({ }^{\prime \prime}{ }^{\prime \prime}\right)\end{array}$ & References \\
\hline HRC-I & 053747.36 & -691020.4 & Wang et al. (2001) \\
HRC-I & 053747.34 & -691019.8 & Gotthelf (p.c.) \\
ACIS-I & 053747.46 & -691019.9 & Townsley et al. (2005) \\
ACIS-S & 053747.42 & -691020.2 & present work \\
\hline
\end{tabular}

ACIS-S frame onto the ACIS-I one. As the effects on the absolute astrometry are roughly of the same magnitude, we assume that the global errors on the ACIS-S positions are similar to the ACIS-I one (about 1.'0).

The available estimates for the Chandra position of PSR J0537-6910 (Table 1) are all consistent within the computed errors. Figure 1 shows the HRC and ACIS pulsar positions superimposed on the ACS/WFC $814 \mathrm{~W}$ image after astrometric recalibration. A few objects (numbered 1 through 12) can be identified within or close to the error circles. The three candidates already investigated by Mignani et al. (2000) are highlighted in Fig. 1 (see caption). Because of their faintness and proximity to brighter stars, all the other objects identified within the error circles could not be detected in the less deep and more confused ground-based NTT observations by Mignani et al. (2000).

\subsection{ACS photometry}

To perform accurate photometry on the ACS images, we run an object detection program using the ROMAFOT package for photometry in crowded fields (Buonanno \& Iannicola 1989). The ROMAFOT parameters were tuned to achieve a $\geq 5 \sigma$ object detection in each filter. A template PSF was obtained by fitting the intensity profiles of some of the brightest, unsaturated, isolated stars in the field with a Moffat function, plus a numerical map of the residual to better take into account the contribution of the PSF wings. As a reference for object detection, we used our $814 \mathrm{~W}$-band image, for which the effects of the local absorption are minimized. The master list of objects was then registered on the images taken in the $435 \mathrm{~W}$ and $555 \mathrm{~W}$ filters and used as a reference for the fitting procedure. A careful check by eye was performed to ensure that all the stellar objects found in the $814 \mathrm{~W}$ band were successfully fitted in the other images and to filter out spurious detections. Aperture corrections were computed by fitting the growth curve of a suitable set of standard stars. Instrumental magnitudes were then converted into the STMAG ${ }^{3}$ photometric system by applying the updated ACS zero points given by De Marchi et al. (2004).

\footnotetext{
3 http://www.stsci.edu/hst/acs/analysis/ zeropoints/\#zeropoints
}

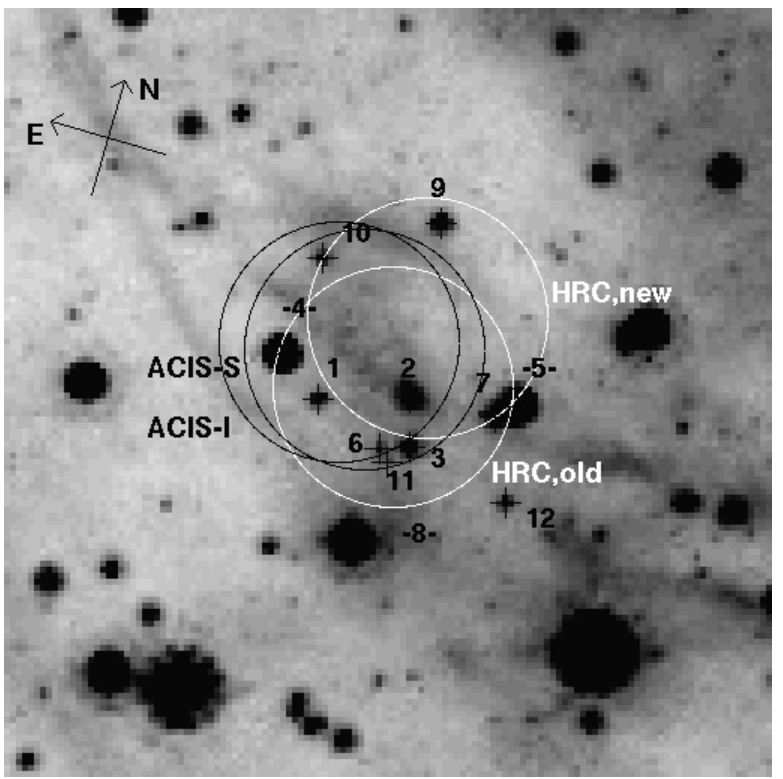

Fig. 1. Field around PSR J0537-6910 (6.'3 × 6.'3) imaged with the ACS filter $814 W$ ( $2800 \mathrm{~s}$ exposure). The four circles indicate the X-ray positions of the pulsar (see Table 1) as derived from observations performed with the Chandra HRC (white; "old" from Wang et al. 2001; "new" from Gotthelf 2003, private communication) and ACIS (black; Townsley et al. 2005). For each circle, the radius corresponds to the overall uncertainty of the pulsar position resulting from the combination of the estimated error of the Chandra coordinates $\left(\sim 1^{\prime \prime}\right)$ and the ACS astrometric solution ( $0{ }^{\prime}$ ' 1 ). Stars 4, 5 and 8 (marked by horizontal ticks) are stars 6, 3 and 4 of Mignani et al. (2000).

\section{Results}

The observed magnitudes and colors of all the potential counterparts identified in Fig. 1 are summarized in Table 2. No other objects were identified within or close to the Chandra error circles down to $5 \sigma$ limiting magnitudes of about 26.4, 26.2, and 27.2 in the $435 \mathrm{~W}, 555 \mathrm{~W}$, and $814 \mathrm{~W}$ passbands, respectively.

We note that the photometry of star 7 is likely affected by an uncertainty larger than the attached formal errors as it is partially blended with the brighter star 5 . The same is probably true also for object 2 which might actually be a doublet of unresolved objects, as suggested by its slightly elongated shape. However, our PSF analysis is affected by the presence of a relatively bright filament crossing the object 2 position. Objects 1, 6, 11 and 12 have been clearly detected only in the $814 \mathrm{~W}$ passband and are probably very reddened. The interstellar extinction toward PSR J0537-6910 is relatively uncertain.Taking as a reference the measurements of the Balmer decrement around N157B (Caplan \& Deharveng 1985), we can derive a color index $E(B-V)=0.32$. On the other hand, from the X-ray spectral fits to the ACIS data of PSR J0537-6910 we derive a hydrogen column density $N_{\mathrm{H}} \sim(0.5-1.0) \times 10^{22} \mathrm{~cm}^{-2}$, depending on the assumptions on the metal abundance (see Sect. 5). By adopting the extinction law appropriate for the 30 Doradus region (Fitzpatrick 1986), these values correspond to $E(B-V)=0.22-0.42$. This gives an average $E(B-V)$ consistent with that derived from Caplan \& Deharveng (1985). 

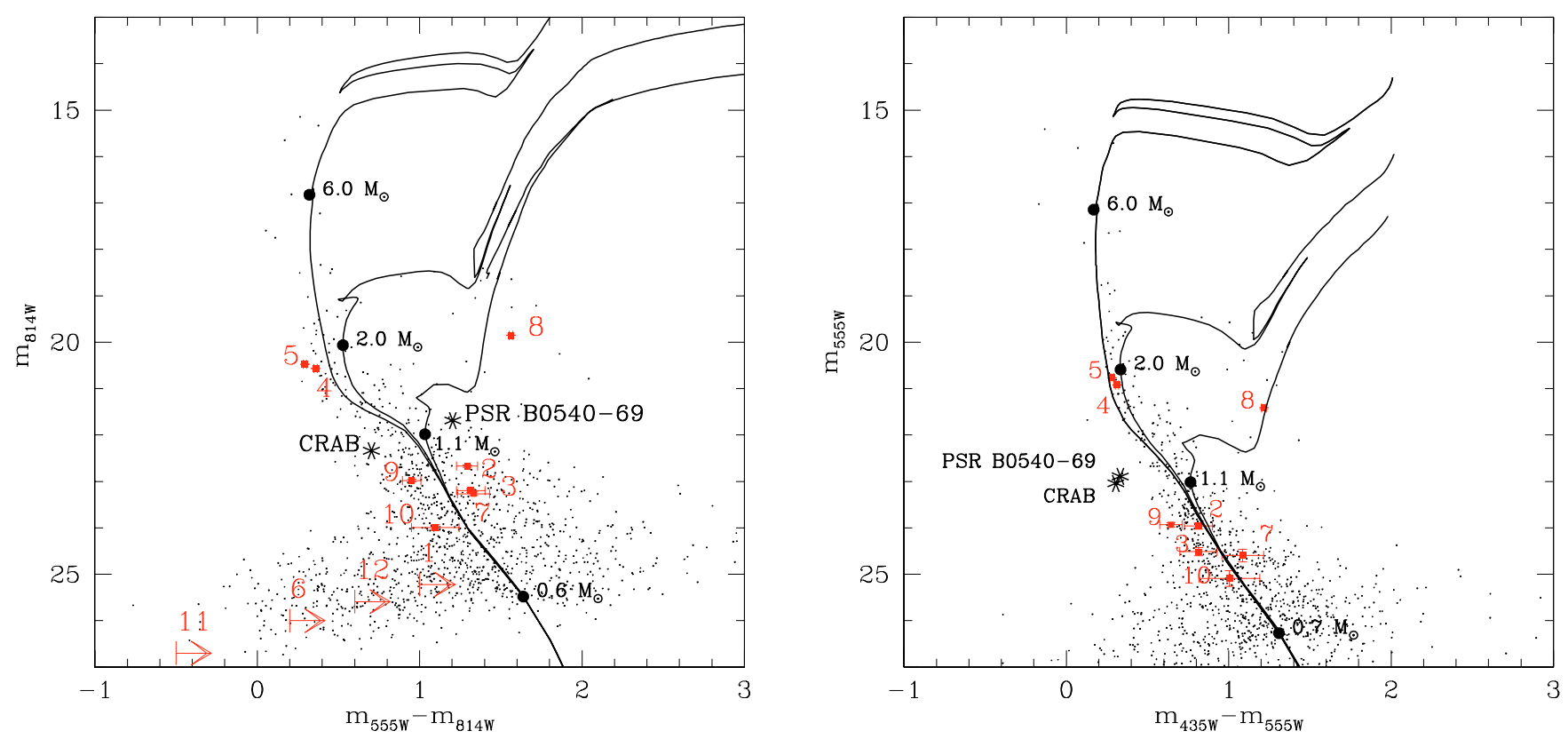

Fig. 2. Observed Color-Magnitude Diagrams (CMDs) of all the stars detected at $\geq 5 \sigma$ significance within a $40^{\prime \prime} \times 40^{\prime \prime}$ box centered on the pulsar position. Field stars are marked by small dots. Objects 2 through 5 and 7 through 10 (Fig. 1) are marked by filled squares and numbered accordingly. In the left diagram, the arrows indicate the limits on the locations of objects 1, 6, 11 and 12. As a reference, the locations of the Crab and PSR B0540-69 are also plotted (asterisks) after renormalizing the fluxes to the N157B distance and extinction. The lines indicate stellar isochrones computed from theoretical models (Salasnich et al. 2000) for different age values (starting from the top: $60 \mathrm{Myr}, 600 \mathrm{Myr}$, and $3 \mathrm{Gyr}$, respectively) and reddened for an assumed $E(B-V)=0.32$ (see text). The filled circles indicate the locations of stars at the Turn-off $\left(1.1,2\right.$ and $\left.6 M_{\odot}\right)$ and of stars close to the detection limit in the $814 \mathrm{~W}$ and $555 \mathrm{~W}$ passbands $\left(0.6\right.$ and $0.7 M_{\odot}$, respectively).

Table 2. Observed magnitudes, colors and associated errors (in parentheses) for all the objects identified in Fig. 1 which have been detected at $\mathrm{a} \geq 5 \sigma$ level in at least one passband. Magnitudes and colors are computed in the STMAG ${ }^{3}$ photometric system.

\begin{tabular}{cccc}
\hline \hline ID & $m_{814 W}$ & $m_{555 W}-m_{814 W}$ & $m_{435 W}-m_{555 W}$ \\
\hline 1 & $25.22(0.04)$ & $\geq+1.0$ & - \\
2 & $23.96(0.02)$ & $-0.04(0.07)$ & $+0.24(0.09)$ \\
3 & $24.49(0.03)$ & $-0.01(0.09)$ & $+0.24(0.12)$ \\
4 & $21.85(0.01)$ & $-0.97(0.01)$ & $-0.26(0.01)$ \\
5 & $21.77(0.01)$ & $-1.03(0.01)$ & $-0.29(0.01)$ \\
6 & $26.00(0.11)$ & $\geq+0.2$ & - \\
7 & $24.55(0.04)$ & $0.00(0.10)$ & $+0.51(0.17)$ \\
8 & $21.14(0.01)$ & $+0.23(0.01)$ & $+0.64(0.02)$ \\
9 & $24.27(0.03)$ & $-0.38(0.06)$ & $+0.07(0.07)$ \\
10 & $25.28(0.06)$ & $-0.23(0.15)$ & $+0.43(0.22)$ \\
11 & $26.70(0.21)$ & $\geq-0.5$ & - \\
12 & $25.59(0.08)$ & $\geq+0.6$ & - \\
\hline
\end{tabular}

For this reason, we will use $E(B-V)=0.32$ as an indicative value, with an uncertainty of \pm 0.1 .

To assess the nature of the candidates listed in Table 2, we compare their locations in the $814 W$ vs. $555 W-814 W$ and $555 W$ vs. $435 W-555 W$ Color-Magnitude Diagrams (CMDs) with those derived from the photometry of a representative number of field stars. Figure 2 shows the CMDs built for all the stars detected at $\geq 5 \sigma$ in a $40^{\prime \prime} \times 40^{\prime \prime}$ region centered on the pulsar position, including objects 2 through 5 and 7 through 10 which were detected in all the passbands (Table 2). For convenience, the computed magnitudes and colors are renormalized to the VEGAMAG ${ }^{3}$ photometric system because it is closer to the Johnson-Cousins system. The conversion from the STMAG to VEGAMAG is done by applying zero-point offsets of $0.61,0.04$, and -1.29 magnitudes for the $435 \mathrm{~W}, 555 \mathrm{~W}$, and $814 W$ filters, respectively (De Marchi et al. 2004). As a reference, we overplot a set of stellar isochrones computed from theoretical models of metallicity $Z=0.008$ and helium abundance $Y=0.23$ (Salasnich et al. 2000) for different age values (60 Myr, $600 \mathrm{Myr}$ and $3 \mathrm{Gyr}$ ). The assumed stellar masses range from $6 M_{\odot}$ for the stars close to the Turn-off of the $60 \mathrm{Myr}$ isochrone, to $\sim 0.6 M_{\odot}$ for the faintest detected objects. For a direct comparison with the observed points, an interstellar extinction corresponding to the assumed $E(B-V)=0.32$ has been applied to the isochrones. Most of the objects detected close to the pulsar position lie along the Main Sequence (MS). The only exception is object 8 (star 4 of Mignani et al. 2000) that lies on the giant branch of the $3 \mathrm{Gyr}$ isochrone.

Thus, none of these objects is distinguishable from the local LMC stellar population and can be identified as a pulsar counterpart based on the observed colors. Unfortunately, very little can be said about the nature of objects 1, 6, 11 and 12 . Based on the $555 \mathrm{~W}-814 \mathrm{~W}$ lower limits (Fig. 2), only object 11 (and perhaps 6) might be substantially bluer compared to the MS and field stars, but this can only be verified with deeper observations. 


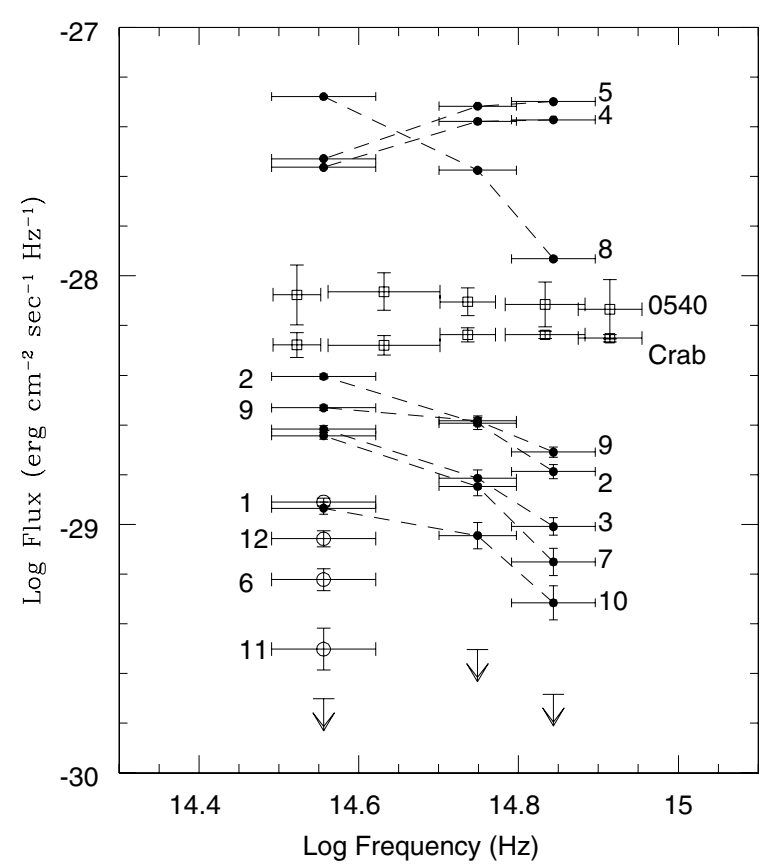

Fig. 3. Spectral energy distributions (SEDs) for the candidate counterparts detected in the three passbands (filled circles) and the $814 \mathrm{~W}$ spectral fluxes for the objects detected in this passband only (open circles). Detection limits for the three filters are indicated. The spectral fluxes were computed at the central wavelengths of the ACS passbands and plotted after correction for an interstellar extinction of $E(B-V)=0.32$, with $A_{814 W}=1.98 E(B-V) ; A_{555 W}=3.41 E(B-V)$ and $A_{435 W}=4.12 E(B-V)$. The numbers correspond to the identifications assigned in Fig. 1. For comparison we have plotted the extinction-corrected SEDs of the Crab and PSR B0540-69 (empty squares), reproduced from Mignani \& Caraveo (2001), after normalization to the N157B distance $(47 \mathrm{kpc})$.

To assess the spectral energy distributions (SEDs) of the candidates, we computed their extinction-corrected spectral fluxes at the central wavelengths of the ACS filters. They are plotted in Fig. 3, together with the $5 \sigma$ upper limits and the extinction-corrected spectral fluxes of the young pulsars Crab and PSR B0540-69 (Mignani \& Caraveo 2001), normalized to the N157B distance. For objects 1, 6, 11 and 12, we plotted only the $814 \mathrm{~W}$ spectral fluxes. We see that the SEDs for most of the objects detected in all the three passbands are markedly different from the flattish spectra of the young Crab and PSR B0540-69. This is certainly the case for objects 4, 5 and 8 , and very likely also for objects 3,7 and 10 , even when accounting for the uncertainty of the interstellar extinction correction. Thus, we conclude that these objects are most likely LMC field stars, in agreement with the results of our CMD analysis, and as such can be reasonably ruled out as potential counterparts to PSR J0537-6910. The only possible exceptions are object 9, which exhibits a flatter SED than the others, and, perhaps, object 2 . However, since the latter might actually be a blend of two unresolved objects (see above), its SED is probably affected by an unknown uncertainty. For objects 1, 6, 11 and 12 , the SEDs are poorly constrained. However, we note that for objects 1,12 and 6 the $555 \mathrm{~W}$ and $435 \mathrm{~W}$ upper limits indicate rather steep spectra suggesting that, whatever is their nature, they can hardly be associated with the pulsar. Only for object 11 the constraints on the SED do not allow us to exclude a flat spectrum.

\section{Discussion}

The results of our multicolor photometry suggest that only two objects, 9 and 2, of the eight detected in more than one filter can be considered as possible optical counterparts to the pulsar, although object 9 is among the most distant from the revised Chandra pulsar position, and object 2 might be a doublet. Of the others, object 11 is the only possible candidate. If either object 9 or object 2, comparable in brightness, were the optical counterpart of PSR J0537-6910, it would have an extinctioncorrected luminosity $L_{\mathrm{opt}} \approx 9 \times 10^{32}$ or $1.2 \times 10^{33} \mathrm{erg} \mathrm{s}^{-1}$ in the $814 \mathrm{~W}$ passband, for an assumed pulsar distance of $47 \mathrm{kpc}$ (Gould 1995). This would imply that the pulsar has an optical emission efficiency $\eta_{\mathrm{opt}} \equiv L_{\mathrm{opt}} / \dot{E} \approx 1.9 \times 10^{-6}$ or $2.4 \times 10^{-6}$. The values of the luminosity are slightly below those of the young pulsars Crab and PSR B0540-69, $L_{\mathrm{opt}} \approx 1.5 \times 10^{33}$ and $\approx 3 \times 10^{33} \mathrm{erg} \mathrm{s}^{-1}$, respectively, normalized to the $814 \mathrm{~W}$ passband. On the other hand, the efficiency would be still comparable with that of the $\operatorname{Crab}\left(\eta_{\mathrm{opt}} \approx 3.4 \times 10^{-6}\right)$ but definitely below the efficiency of PSR B0540-69 $\left(\eta_{\mathrm{opt}} \approx 2 \times 10^{-5}\right)$ which is by far the most efficient optical pulsar to date (see, e.g., Zharikov et al. 2004).

If, instead, object 11 were the pulsar counterpart, it would be fainter by a factor of about 10 in the optical, with an extinction-corrected luminosity $L_{\mathrm{opt}} \approx 9 \times 10^{31} \mathrm{erg} \mathrm{s}^{-1}$ and an efficiency $\eta_{\mathrm{opt}} \approx 1.9 \times 10^{-7}$. Finally, if none of the present candidates were the counterpart, the measured $814 \mathrm{~W}$ upper limit would imply an optical luminosity $L_{\mathrm{opt}} \leq 5.7 \times 10^{31} \mathrm{erg} \mathrm{s}^{-1}$ and a corresponding efficiency $\eta_{\text {opt }} \leq 1.2 \times 10^{-7}$. In both cases, PSR J0537-6910 would be significantly underluminous with respect to the Crab and PSR B0540-69.

We note that including the uncertainty on the optical extinction correction affects the above estimates of $L_{\mathrm{opt}}$ and $\eta_{\mathrm{opt}}$ by $\leq 20 \%$ (in the $814 \mathrm{~W}$ filter) and does not alter significantly our conclusions.

It is also interesting to confront the results of the optical observations with the X-ray data on PSR J0537-6910. We analyzed the 30-ks Chandra ACIS-S3 observation of the pulsar, taken in $1 / 4$ subarray mode (frame time $0.8 \mathrm{~s}$ ). After correcting for pile-up (pile-up fraction was about 5\%-6\%) and subtracting the contribution from the bright PWN, we obtained a powerlaw fit of the pulsar spectrum with a photon index $\Gamma=1.8 \pm 0.1$ and a photon spectral flux $\mathcal{N}=(4.4 \pm 0.7) \times 10^{-4}$ photons $\mathrm{cm}^{-2} \mathrm{~s}^{-1} \mathrm{keV}^{-1}$ at $E=1 \mathrm{keV}$. These values are almost independent of the assumed metal abundance in the absorbing (LMC) ISM, contrary to the hydrogen column density: $N_{\mathrm{H}}=(0.95 \pm 0.07) \times 10^{22} \mathrm{~cm}^{-2}$ for metal abundances $40 \%$ of solar, and $N_{\mathrm{H}}=(0.50 \pm 0.05) \times 10^{22} \mathrm{~cm}^{-2}$ for solar abundances. The derived spectral parameters correspond to the X-ray luminosity $L_{\mathrm{X}}=(5.4 \pm 1.1) \times 10^{35} \mathrm{erg} \mathrm{s}^{-1}$ and X-ray efficiency $\eta_{\mathrm{X}} \equiv L_{\mathrm{X}} / \dot{E} \approx 1.1 \times 10^{-3}$, in the $1-10 \mathrm{keV}$ band, significantly below those of the Crab $\left(L_{\mathrm{X}}=1.5 \times 10^{36} \mathrm{erg} \mathrm{s}^{-1}\right.$, $\left.\eta_{\mathrm{X}}=3.4 \times 10^{-3}\right)$ and PSR B0540-69 $\left(L_{\mathrm{X}}=2.4 \times 10^{36} \mathrm{erg} \mathrm{s}^{-1}\right.$, $\eta_{\mathrm{X}}=1.6 \times 10^{-2} ;$ see Zavlin \& Pavlov 2004). 


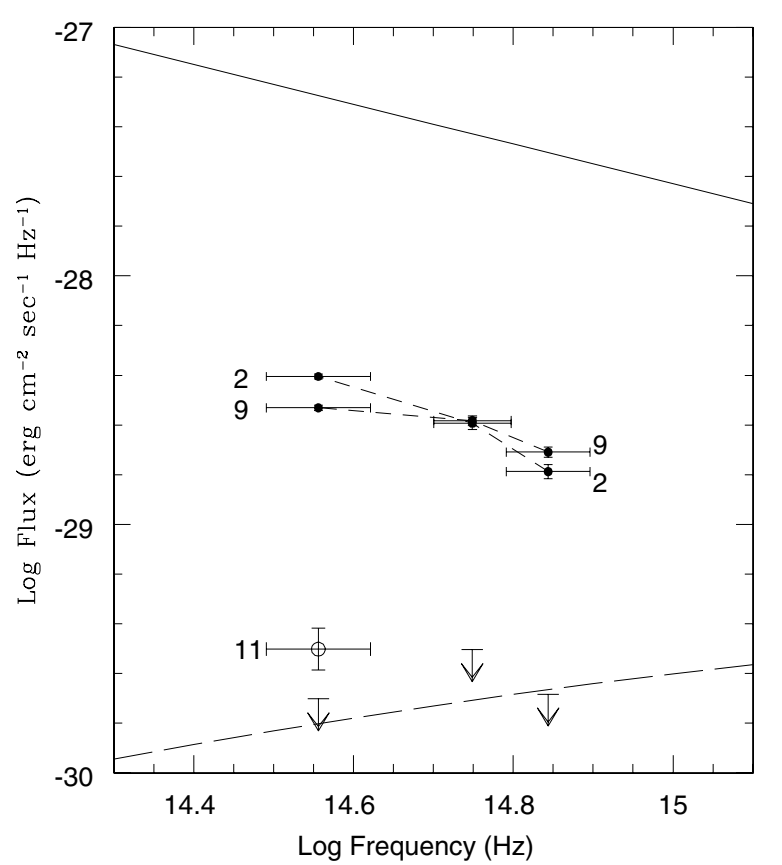

Fig. 4. Comparison between the optical SEDs of objects 2, 9 and 11 and the extrapolations into the optical domain of the power-law (solid) and curved power-law (dashed) models best fitting the Chandra and BeppoSAX/RXTE X-ray spectra, respectively (see text).

While the X-ray and optical efficiencies show very large scatter among different pulsars, up to at least 3 orders of magnitude, their ratio is much more stable, typically $\eta_{\mathrm{opt}} / \eta_{\mathrm{X}}=$ $L_{\text {opt }} / L_{\mathrm{X}} \sim 1 \times 10^{-3}$ (for the optical and X-ray luminosities measured in the $814 \mathrm{~W}$ and $1-10 \mathrm{keV}$ bands, respectively), which suggests a common mechanism for the magnetospheric X-ray and optical emission (Zavlin \& Pavlov 2004). If object 9 (or 2) is the counterpart of PSR J0537-6910, this ratio, $L_{\text {opt }} / L_{\mathrm{X}} \approx 1.6 \times 10^{-3}$ (or $2.2 \times 10^{-3}$ ), would be comparable with the average one for optically detected pulsars.

On the other hand, if object 11 is the pulsar counterpart (or the counterpart is even fainter than our detection limit), then the optical-to-X-ray luminosity ratio becomes $\approx 1 \times 10^{-4}$, i.e. the lowest among all pulsars detected in the optical, which strengthens the case for object 9 or 2 being the pulsar counterpart.

For additional comparison of the optical and X-ray data on PSR J0537-6910, we show in Fig. 4 extrapolations of the pulsar's X-ray spectrum into the optical and the optical spectral fluxes for three putative candidates. We see that the extrapolation of the power-law fit into the optical exceeds the fluxes of the brighter candidates (objects 9 and 2) by a factor of 10-30, which suggests that the broad-band (optical through X-ray) spectrum cannot be described by a simple power law. A similar flattening of the spectrum with decreasing frequency has been observed for other young pulsars (e.g., Mineo et al. 2003). It can be described with a curved power law: $f_{E}=\mathcal{N} E^{-\Gamma(E)}$, where the simplest form of the energy-dependent photon in$\operatorname{dex}$ is $\Gamma(E)=a+b \log E$ ( $f_{E}$ is the photon spectrum, $E$ the photon energy in $\mathrm{keV})$. In addition to the simple powerlaw fit $(b=0, a=\Gamma)$, we show in Fig. 4 a curved powerlaw fit $(a=1.33 \pm 0.12, b=0.15 \pm 0.05)$ obtained by
Mineo et al. (2003) for the pulsed X-ray spectrum of PSR J0537-6910 observed with BeppoSAX and RXTE in the 1-30 keV band, where we have scaled the normalization to the Chandra value to account for the unpulsed component. Extrapolation of this fit into the optical predicts optical fluxes comparable to the limits of our observation, well below the fluxes of objects 9 and 2. However, the example of the Crab pulsar (Massaro et al. 2000) shows that such an extrapolation may underpredict the optical fluxes by an order of magnitude. Therefore, the comparison with the X-ray spectrum of PSR J0537-6910 suggests that one of our three candidates is the pulsar's optical counterpart, unless the PSR J0537-6910 is unusually underluminous in the optical.

\section{Conclusions}

We have performed deep, high-resolution, multicolor observations of the PSR J0537-6910 field with the ACS/WFC on board HST. Two likely candidate counterparts, compatible with the revised Chandra position of the pulsar, have been identified based on the color information. Although these candidates look interesting for their spectra, the still limited multicolor information, as well as the uncertainty of the interstellar extinction toward the pulsar, do not allow yet to obtain a compelling spectral characterization. For the third, fainter, candidate, detected in one filter only, the spectrum is virtually unconstrained. Thus, it is not possible to determine yet which of the three, if any, is indeed the actual pulsar counterpart. A follow-up timing observation will allow a more in-depth investigation of at least the two brighter candidates and, hopefully, will provide the evidence required to finally establish the optical identification of the pulsar.

Acknowledgements. We are indebted to Eric Gotthelf for providing the revised HRC coordinates of the pulsar. It is a pleasure to thank Guido De Marchi for providing us with the ACS photometric calibrations prior to publication. The work of G.G.P. was partially supported by NASA grant NAG5-10865.

\section{References}

Alexander, D. M., Bauer, F. E., Brandt, W. N., \& Schneider 2003, AJ, 126,539

Buonanno, R., \& Iannicola, G. 1989, PASP, 101, 294

Butler, R. F., Golden, A., Shearer, A., \& Gouiffes, C. 2002, Proc. 270, WE-Heraeus Seminar on Neutron Stars, Pulsars, and Supernova Remnants, ed. W. Becker, H. Lesch, \& J. Trümper, MPE Report, 278

Caplan, J., \& Deharveng, L. 1985, A\&AS, 62, 63

Crawford, F., Kaspi, V. M., Manchester, R. N., et al. 1998, MemSAIt, 69, 951

Cusumano, G., Maccarone, M. C., Mineo, T., et al. 1998, A\&A, 333, L55

De Marchi, G., Sirianni, M., Gilliland, R., \& the ACS team 2004, Instrument Science Report ACS 04-06 (Baltimore: Space Telescope Science Institute)

Fitzpatrick, E. L. 1986, AJ, 92, 1068

Getman, K. V., Flaccomio, E., Broos, P. S., et al. 2005, ApJS, in press [arXiv:astro-ph/0410136] 
Gouiffes, K., \& Ögelman, H. 2000, Proc. IAU Coll. 177, Pulsar Astronomy - 2000 and Beyond, ed. M. Kramer, N. Wex, \& R. Wielebinski, ASP Conf. Ser., 301

Gould, A. 1995, ApJ, 452, 189

Lasker, B., Sturch, C. R., McLean, B. J., et al. 1990, AJ, 99, 2019

Marshall, F. E., Gotthelf, E. V., Zhang, W., et al. 1998, ApJ, 499, L179

Marshall, F. E., Gotthelf, E. V., Middleditch, J., Wang, Q. D., \& Zhang, W. 2004, ApJ, 603, 682

Massaro, E., Cusumano, G., Litterio, M., \& Mineo, T. 2000, A\&A, 361,695

Middleditch, J., Marshall, F. E., Zhang, W., Gotthelf, E. V., \& Wang, Q. D. 2001, A\&AS, 32, 36.06

Mignani, R. P., \& Caraveo, P. A. 2001, A\&A, 376, 213

Mignani, R., Pulone, L., Marconi, G., Iannicola, G., \& Caraveo, P. A. 2000, A\&A, 355, 603

Mineo, T., Cusumano, G., \& Massaro, E. 2003, Proc. The Restless High-Energy Universe, ed. E. P. J. van den Heuvel, J. J. M. in't Zand, \& R. A. M. J. Wijers [arXiv: astro-ph/0309523]
Monet, D., Levine, S. E., Canzian, B., et al. 2003, AJ, 125, 984

Pavlovsky, C., Biretta, J., Boffi, F., et al. 2003, ACS Instrument Handbook, Version 4.0 (Baltimore, STScI)

Salasnich, B., Girardi, L., Weiss, A., \& Chiosi, C. 2000, A\&A, 361, 1023

Skrutskie, M. F., Schneider, S. E., Stiening, R., et al. 1997, Proc. The Impact of Large Scale Near-IR Sky Survey, ed. F. Garzon et al. (Dordrecht: Kluwer), 25

Townsley, L., et al. 2005, in preparation

Wang, Q. D., \& Gotthelf, E. V. 1998, ApJ, 509, L109

Wang, Q. D., Gotthelf, E. V., Chu, Y. N., \& Dickel, J. R. 2001, ApJ, 559,275

Zacharias, N., Urban, S. E., Zacharias, M. I., et al. 2004, AJ, 127, 3043

Zavlin, V. E., \& Pavlov, G. G. 2004, ApJ, 616, 452

Zharikov, S. V., Shibanov, Yu. A., Mennickent, R. E., et al. 2004, A\&A, 417, 1017 